\title{
Riemann surfaces and restrictively-marked hypermaps
}

\author{
Antonio Breda d'Azevedo * \\ University of Aveiro, 3810-193 Aveiro, Portugal
}

Received 30 September 2007, accepted 17 March 2010, published online 9 April 2010

\begin{abstract}
If $\mathcal{S}$ is a compact Riemann surface of genus $g>1$, then $\mathcal{S}$ has at most $84(g-1)$ (orientation preserving) automorphisms (Hurwitz). On the other hand, if $G$ is a group of automorphisms of $\mathcal{S}$ and $|G|>24(g-1)$ then $G$ is the automorphism group of a regular oriented map (of genus $g$ ) and if $|G|>12(g-1)$ then $G$ is the automorphism group of a regular oriented hypermap of genus $g$ (Singerman). We generalise these results and prove that if $|G|>g-1$ then $G$ is the automorphism group of a regular restrictedlymarked hypermap of genus $g$. As a special case we also show that a marked finite transitive permutation group (Singerman) is a restrictedly-marked hypermap with the same genus.
\end{abstract}

Keywords: Groups, Riemann surface, hypermaps, maps, restrictedly-marked, restrictedly regular.

Math. Subj. Class.: 05C15, 05C10, 30F10

\section{Introduction}

A compact Riemann surface $\mathcal{S}$ of genus $g>1$ has at most $84(g-1)$ orientation-preserving automorphisms (Hurwitz [5]). On the other hand, if a group $G$ of automorphisms of a compact Riemann surface $\mathcal{S}$ of genus $g$ is sufficiently large, then it represents the automorphism group of a regular oriented map (if $|G|>24(g-1)$ ) or hypermap (if $|G|>$ $12(g-1))$, (Singerman [8]). Hypermaps can be seen as restrictedly-marked maps, in this case restricted to the subgroup $\Upsilon^{\hat{0}}=\left\langle R_{1}, R_{2}, R_{1}^{R_{0}}\right\rangle \cong C_{2} * C_{2} * C_{2}$ of index 2 in $\Upsilon=\left\langle R_{0}, R_{1}, R_{2} \mid R_{i}^{2}=\left(R_{2} R_{0}\right)^{2}=1\right\rangle$ (Jones and Breda [2]). As such they are represented by bipartite-maps (2-coloured maps called Walsh bipartite maps) where the hypermap's automorphisms (similarly for homomorphisms) are bipartition-preserving map's automorphisms. Orientable maps $\left(\Upsilon^{+}\right.$-conservative maps) give rise to the restricted forms

\footnotetext{
* Research partially supported by UI\&D "Matemática e Aplicações" of Universidade de Aveiro through the Program POCTI of FCT, cofinanced by the European Community fund FEDER.

E-mail address: breda@ua.pt (Antonio Breda d'Azevedo)
} 
known as "oriented" maps $\left(\Upsilon^{+}\right.$-marked maps), described by triples $(\Omega, R, L)$ consisting of a finite set $\Omega$ and two $\Omega$-permutations $R$ and $L$, with $L^{2}=1$, generating a transitive group on $\Omega$. Oriented hypermaps $\left(\Delta^{+}\right.$-marked hypermaps), the restricted forms of $\Delta^{+}$conservative (or orientable) hypermaps, are direct generalisations of that. More generally yet are the restrictedly-marked hypermaps, the restricted forms of " $\Theta$-conservative" hypermaps, where $\Theta$ is a normal subgroup of finite index of $\Delta=C_{2} * C_{2} * C_{2}$. Often these result in "multi-coloured" maps.

In this paper we generalise Singerman's results and prove that if $G$ is a group of automorphisms of a compact Riemann surface of genus $g>1$, and $|G|>g-1$, then $G$ is the automorphism group of a regular restrictedly-marked hypermap of genus $g$.

\subsection{Regular restrictedly-marked hypermaps}

Algebraically, hypermaps correspond to finite transitive permutations representations $\nu$ : $\Delta \longrightarrow G, R_{i} \mapsto r_{i}$, where $\Delta$ is the free product $C_{2} * C_{2} * C_{2}$ generated by the reflections in the sides of a hyperbolic triangle with zero internal angles in the hyperbolic plane. Let $\Theta$ be a normal subgroup of finite index $n$. A hypermap $\mathcal{H}$ is $\Theta$-conservative if its fundamental subgroup $H$ is a subgroup of $\Theta$. In such case $\Theta$ acts on its flags uniformly dividing them into $\Theta$-orbits of equal length. A $\Theta$-conservative hypermap $\mathcal{H}$ is $\Theta$-regular if and only if the group $A u t^{\Theta}(\mathcal{H})$, of the automorphisms of $\mathcal{H}$ preserving each $\Theta$-orbit, acts transitively on each $\Theta$-orbit; and this happens if and only if $H$ is normal in $\Theta$. A hypermap is restrictedlymarked if it is $\Theta$-conservative, and is restrictedly-regular if it is $\Theta$-regular, for some normal subgroup $\Theta$ of finite index in $\Delta$. Not every hypermap is restrictedly-regular, see [1].

By the Kurosh Subgroup Theorem (see [7], Corollary 4.9.1 and remarks after Corollary 4.9.2), $\Theta$ freely decomposes uniquely (up to a permutation of factors) in a free product $C_{2} * \cdots * C_{2} * C_{\infty} * \cdots * C_{\infty}=\left\langle X_{1}, \ldots, X_{m} \mid X_{i}^{2}=1, i=1 \ldots s\right\rangle$, for some $0 \leq s \leq m$. A $\Theta$-conservative hypermap is combinatorially described by a $(m+1)$-tuple

$$
\mathcal{Q}=\left(\Omega ; x_{1}, \ldots, x_{m}\right)
$$

where $\Omega$ is a finite set (the set of the " $\Theta$-slices"), $x_{1}, \ldots, x_{m}$ are permutations of $\Omega$ generating a group $G$ acting transitively on $\Omega$ such that the function $\rho: X_{i} \mapsto x_{i}$ extends to an epimorphism from $\Theta$ to $G$. Such $(m+1)$-tuple is called a $\Theta$-marked hypermap. For example, if $\Theta$ is the even-word subgroup $\Delta^{+} \cong F(2)$, a $\Delta^{+}$-conservative (i.e. orientable) hypermap is described by a $\Delta^{+}$-marked (i.e. oriented) hypermap $\mathcal{Q}=\left(\Omega ; x_{1}, x_{2}\right)$, where $x_{1}, x_{2}$ are usually denoted by the letters $R, L$ or by $\rho, \lambda$. On the other hand, if $Q$ is the fundamental $\Theta$-marked subgroup of $\mathcal{Q}$, i.e. the stabiliser of some fixed $\omega \in \Omega$ under the action of $\Theta$ on $\Omega$, then the " $\Delta$-form" hypermap ${ }^{\Delta} \mathcal{Q}=\left(\Delta / r Q ; Q_{\Delta} R_{0}, Q_{\Delta} R_{1}, Q_{\Delta} R_{2}\right)$, where $Q_{\Delta}$ is the core of $Q$ in $\Delta$ and $\Delta / r Q$ is the set of the right cosets of $Q$ in $\Delta$, is $\Theta$-conservative and shares with $\mathcal{Q}$ the same underlying surface, the same underlying hypergraph, the same set $\mathcal{V}$ of hypervertices, the same set $\mathcal{E}$ of hyperedges and the same set $\mathcal{F}$ of hyperfaces; only the set $\Omega$ of $\Theta$-slices of $\mathcal{Q}$ is just an orbit of the action of $\Theta$ on the set of flags $F=\Delta /_{r} Q$ of ${ }^{\Delta} \mathcal{Q}$. The sizes of $\Omega$ and $F$ are $|\Theta: Q|$ and $|\Delta: Q|$ respectively. The permutations $x_{1}, \ldots, x_{m}$ are restrictions to $\Omega$ of the permutations $X_{1} \nu, \ldots, X_{m} \nu \in \operatorname{Sym}(F)$, where $\nu: \Delta \longrightarrow \operatorname{Mon}\left({ }^{\Delta} \mathcal{Q}\right)$ is the canonical transitive permutation representation. A $\Theta$-marked hypermap $\mathcal{Q}$ is regular if its $\Delta$-form ${ }^{\Delta} \mathcal{Q}$ is $\Theta$-regular. Moreover, $\mathcal{Q}$ has boundary if ${ }^{\Delta} \mathcal{Q}$ has boundary. That is, if $R_{i} \in Q^{d}$ for some $d \in \Delta$ and $i=0,1,2$. If $\mathcal{Q}$ has no boundary, then the Euler characteristic of $\mathcal{Q}$ (we mean the Euler characteristic of its underlying surface, 
which is the Euler characteristic of ${ }^{\Delta} \mathcal{Q}$ ) is given by $\chi(\mathcal{Q})=|\mathcal{V}|+|\mathcal{E}|+|\mathcal{F}|-\frac{|F|}{2}$, where $|F|=n|\Omega|$.

Let $(k ; l ; m)$ be the type of the trivial $\Theta$-marked hypermap $\mathcal{T}_{\Theta}=\left(\Theta / \Theta ; \Theta X_{1}, \ldots\right.$, $\left.\Theta X_{m}\right)$, with just one $\Theta$-slice; geometrically a $\Theta$-slice is the connected (polygonal) region obtained by the elements of a fixed Schreier transversal for $\Theta$ in $\Delta$ acting on a single flag (a triangular region). The $\Delta$-form of $\mathcal{T}_{\Theta}$ is the hypermap ${ }^{\Delta} \mathcal{T}_{\Theta}=\left(\Delta / \Theta ; \Theta R_{0}, \Theta R_{1}, \Theta R_{2}\right)$ with $n$ flags. If $\mathcal{Q}$ is a regular $\Theta$-marked hypermap then $\mathcal{Q}$ regularly covers ${ }^{1} \mathcal{T}_{\Theta}$ and, as a consequence, its hypervertices (resp. hyperedges, hyperfaces) will be regularly partitioned (or coloured) in $q_{v}$ parts, each part projecting to a hypervertex (resp. hyperedge, hyperface) of $\mathcal{T}_{\Theta}$. Hypervertices (resp. hyperedges, hyperfaces) of the same colour have the same valency. This induces a sequence

$$
\left(k_{1}, \ldots, k_{q_{v}} ; l_{1}, \ldots, l_{q_{e}} ; m_{1}, \ldots, m_{q_{f}}\right)
$$

called the $\Theta$-type of $\mathcal{Q}$, where $k_{1}, \ldots, k_{q_{v}}$ are the common valencies of the hypervertices in the same coloured-parts $1, \ldots, q_{v}$, respectively, and similarly for the rest of the numbers. Here $q_{v}, q_{e}$ and $q_{f}$ are the numbers of hypervertices, hyperedges and hyperfaces of $\mathcal{T}_{\Theta}$, respectively. The Euler characteristic of $\mathcal{Q}$ is then expressed by ( $\operatorname{cf} \S 9.1$ of [1])

$$
\chi(\mathcal{Q})=|\Omega|\left(\sum_{i=1}^{q_{v}} \frac{\mu_{v} k}{2 k_{i}}+\sum_{i=1}^{q_{e}} \frac{\mu_{e} l}{2 l_{i}}+\sum_{i=1}^{q_{f}} \frac{\mu_{f} m}{2 m_{i}}-\frac{n}{2}\right)
$$

where $\mu_{v}=1$ or 2 according as the hypervertex $v$ of $\mathcal{T}_{\Theta}$ lies on the boundary or not, and similarly for $\mu_{e}$ and $\mu_{f}$. Note that $n$ is the index of $\Theta$ in $\Delta$. For further reading on the subject, and for geometric illustrations, we refer the reader to [1].

\section{Two special restrictedely-marked subgroups}

Example 1. Let $\Theta$ be the subgroup $\Delta_{n}$ in $\Delta$ of index $2 n$ generated by the $n+1$ generators

$$
Z_{1}=R_{1} R_{2}, Z_{2}=\left(R_{1} R_{2}\right)^{R_{0} R_{2}}, \ldots, Z_{n}=\left(R_{1} R_{2}\right)^{\left(R_{0} R_{2}\right)^{n-1}} \text { and } Z_{n+1}=\left(R_{2} R_{0}\right)^{n}
$$

This group is normal in $\Delta=\left\langle R_{0}, R_{1}, R_{2}\right\rangle=\left\langle Z_{1}, R_{0} R_{2}, R_{2}\right\rangle$, for $Z_{i}^{R_{0} R_{2}}=Z_{i+1}, i=$ $1,2, \ldots, n-1, Z_{n}^{R_{0} R_{2}}=Z_{1}^{Z_{n+1}^{-1}}, Z_{n+1}^{R_{0} R_{2}}=Z_{n+1}, Z_{1}^{R_{2}}=Z_{1}^{-1}, Z_{i}^{R_{2}}=\left(Z_{n-(i-2)}^{Z_{n+1}}\right)^{-1}, i=$ $2, \ldots, n$ and $Z_{n+1}^{R_{2}}=Z_{n+1}^{-1}$. The quotient $\Delta / \Delta_{n}$ is a dihedral group $D_{n}$ of order $2 n$. By the Reidmaster-Schreier Rewriting Process, $\Delta_{n}$ is isomorphic to a free product $C_{\infty} *$ $\cdots * C_{\infty}(n+1$ times $)$, that is, a free group of rank $n+1$, and consequently any $\Delta_{n^{-}}$ marked hypermap has the form $\mathcal{Q}=\left(\Omega, z_{1}, \ldots, z_{n+1}\right)$. As the generators lie in $\Delta_{1}=$ $\Delta^{+}=\left\langle R_{1} R_{2}, R_{2} R_{0}\right\rangle$ (the subgroup of the even-length words of $\Delta$ ), any $\Delta_{n}$-marked hypermap is orientable. The trivial $\Delta_{n}$-marked hypermap $\mathcal{T}_{\Delta_{n}}$ is the regular hypermap of type $(1 ; n ; n)$ on the sphere (pictured below for the case when $n=7$ ) with $n$ hypervertices, one hyperedge and one hyperface. Any $\Delta_{n}$-conservative hypermap $\mathcal{H}$ covers $\mathcal{T}_{\Delta_{n}}$ and so its hypervertices are $n$-coloured, which makes $\mathcal{H}$ a $(n+2)$-coloured map.

\footnotetext{
${ }^{1} \mathrm{~A}$ (hyper)map $\mathcal{M}^{\prime}$ with fundamental subgroup $M^{\prime}$ regularly covers $\mathcal{M}$ with fundamental subgroup $M$ if $M^{\prime}$ is a normal subgroup of $M$ (Jones and Singerman [6]).
} 


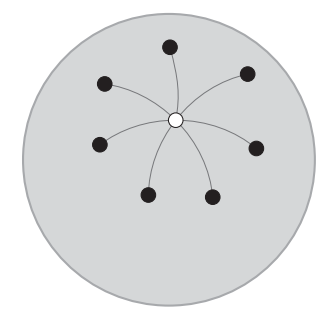

Figure 1: The trivial $\Delta_{7}$-marked hypermap $\mathcal{T}_{\Delta_{7}}$.

The $\Delta_{n}$-type of $\mathcal{Q}$ is therefore $\left(k_{1}, \ldots, k_{n} ; l ; m\right)$ for some positive integers $k_{1}, \ldots, k_{n}$, $l$ and $m$ such that $n$ divides both $l$ and $m$. By (1.2), the Euler characteristic of a regular $\Delta_{n}$-marked hypermap of $\Delta_{n}$-type $\left(k_{1}, \ldots, k_{n} ; l ; m\right)$ is given by

$$
\chi(\mathcal{Q})=|\Omega|\left(\frac{1}{k_{1}}+\cdots+\frac{1}{k_{n}}+\frac{n}{l}+\frac{n}{m}-\frac{2 n}{2}\right) .
$$

If $\mathcal{Q}=\left(\Omega, z_{1}, \ldots, z_{n+1}\right)$ is a $\Delta_{n}$-marked hypermap then each orbit of $z_{i}$, for $i=1, \ldots, n$, corresponds to a $i$-coloured hypervertex $v_{i}$ (denoting the $i$-coloured hypervertices by $v_{i}, w_{i}$, etc.) while each orbit of $z_{n+1}$ corresponds to a hyperedge (a white vertex in the picture below) and each orbit of the product $z_{1} \ldots z_{n+1}$ correspond to a hyperface.

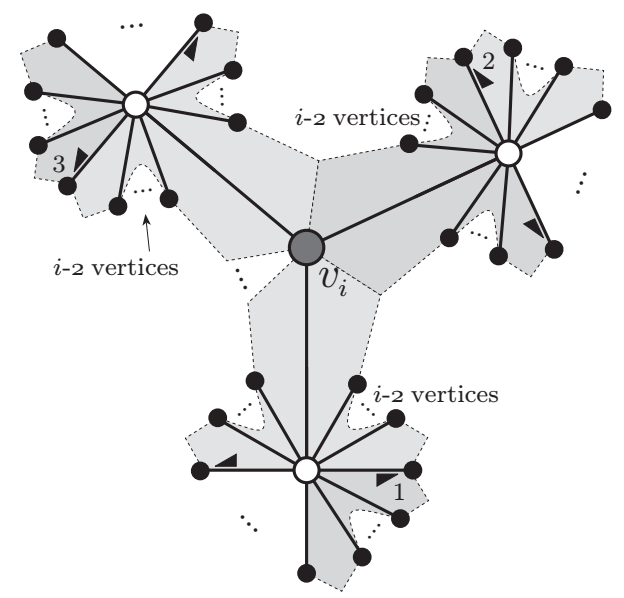

Figure 2: Part of a $z_{i}$-orbit $(i \geq 2)$ labeled $1,2,3, \ldots$

If $\mathcal{Q}$ is regular (equivalently, its $\Delta$-form ${ }^{\Delta} \mathcal{Q}=\left(F, r_{0}, r_{1}, r_{2}\right)$ is $\Delta_{n}$-regular) the automorphism group $\operatorname{Aut}(\mathcal{Q})$, which is the $\Delta_{n}$-automorphism group of its $\Delta$-form ${ }^{\Delta} \mathcal{Q}$, coincides with the monodromy group $\operatorname{Mon}(\mathcal{Q})$ - only the actions of $\operatorname{Mon}(\mathcal{Q})$ and $\operatorname{Aut}(\mathcal{Q})$ on $F$ are different. Hence $A u t(\mathcal{Q})$ is generated by $z_{1}, \ldots, z_{n+1}$ (considered as automorphisms). Each $Z_{i} \in \Delta_{n}, i=1, \ldots, n$, is a conjugate of the product of two of the hyperbolic reflections $R_{0}, R_{1}$ and $R_{2}$, so $Z_{1}, \ldots, Z_{n}$ are (parabolic) limit rotations about hypervertices, $Z_{n+1}$ is a $n$-step limit rotation about a hyperedge and the product $Z_{1} \ldots Z_{n}$ is a $n$-step limit rotation about a hyperface. They project via $\rho$ to automorphisms $z_{i} \in A u t(\mathcal{Q})$. Notice that ${ }^{\Delta} \mathcal{Q}$ may be not regular (that is, $\mathcal{Q}$ may be not $\Delta$-symmetric); if this is the case, 
the size of the automorphism group $\operatorname{Aut}\left({ }^{\Delta} \mathcal{Q}\right)$ is smaller than $|F|$ and consequently at least one of $r_{0}, r_{1}$ and $r_{2}$ cannot be realised as an automorphism of ${ }^{\Delta} \mathcal{Q}$. However, $\operatorname{Aut}\left({ }^{\Delta} \mathcal{Q}\right)$ acts regularly on the $\Delta_{n}$-orbit $\Omega$ and thus $z_{1}, \ldots, z_{n}$ can be realised as automorphisms of ${ }^{\Delta} \mathcal{Q}$. Now $\mathcal{Q}$ has $n$ hypervertex-colours $v_{1}, v_{2}, \ldots, v_{n}$, only one hyperedge-colour $e$ and only one hyperface-colour $f$. These $n$ hypervertex-colours appear around any hyperedge as well as around any hyperface as shown on Figure 3 . The generators $z_{1}, \ldots, z_{n}$, being the projections of $Z_{1}, \ldots, Z_{n}$, are clearly rotations about the hypervertices $v_{1}, \ldots, v_{n}$ of orders $k_{1}, \ldots, k_{n}$ respectively. The generator $z_{n+1}$ is a rotation about $e$ of order $\frac{l}{n}$ and the product $z_{n+2}=z_{1} z_{2} \ldots z_{n+1}=\left(r_{1} r_{0}\right)^{n}$ is a rotation about $f$ of order $\frac{m}{n}$.

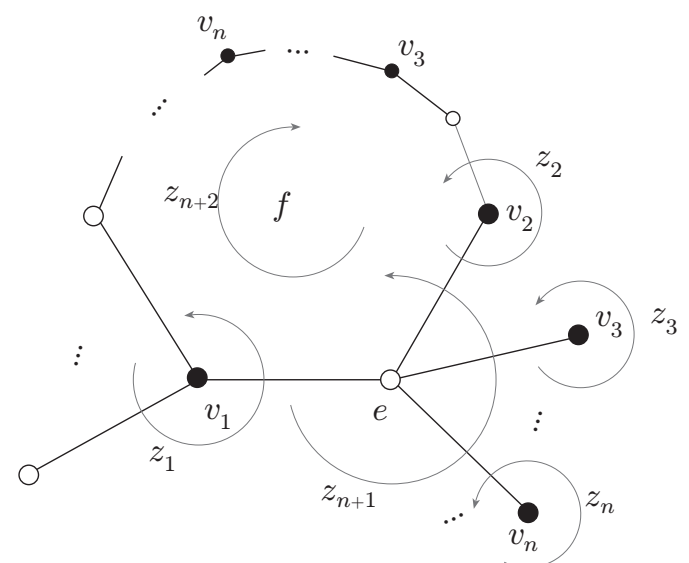

Figure 3: $z_{1}, \ldots, z_{n+2}$ seen as automorphisms of $\mathcal{Q}$.

Formula (2.1) can then be rewritten as follows.

Lemma 2.1. If $\mathcal{Q}=\left(\Omega, z_{1}, \ldots, z_{n+1}\right)$ is a regular $\Delta_{n}$-marked hypermap then the Euler characteristic of $\mathcal{Q}$ is given by

$$
\chi(\mathcal{Q})=|\Omega|\left(\frac{1}{\left|z_{1}\right|}+\cdots+\frac{1}{\left|z_{n}\right|}+\frac{1}{\left|z_{n+1}\right|}+\frac{1}{\left|z_{1} \ldots z_{n+1}\right|}-n\right) .
$$

If $G$ is a group generated by $g_{1}, \ldots, g_{m}$ such that the function $x_{i} \mapsto g_{i}$ extends to an epimorphism from $\Theta$ to $G$, then $\mathcal{Q}=\left(G, g_{1}, \ldots, g_{m}\right)$ is a regular $\Theta$-marked hypermap (Theorem 22 of [1]). A slightly more general statement is obtained by taking the free group $\Theta=\Delta_{m-1}<\Delta^{+}$of rank $m$ :

Lemma 2.2. If $G$ is a group generated by $g_{1}, \ldots, g_{m}$, then $\mathcal{Q}=\left(G, g_{1}, \ldots, g_{m}\right)$ is a regular $\Delta_{m-1}$-marked hypermap, and so, a regular restrictedely-marked hypermap.

Example 2. The subgroup $\Theta=K_{3}$ of $\Delta$ of index 6 generated by $A=R_{0} R_{1}{ }^{R_{2}}, B=$ $R_{0} R_{2}{ }^{R_{1}}, C=R_{1} R_{0}{ }^{R_{2}}$ and $D=R_{1} R_{2}{ }^{R_{0}}$ is normal in $\Delta$, for $A^{R_{0}}=A^{-1}, A^{R_{1}}=D B$, $A^{R_{2}}=C^{-1}, B^{R_{0}}=B^{-1}, B^{R_{1}}=D A, B^{R_{2}}=C^{-1} B^{-1} A, C^{R_{0}}=B D, C^{R_{1}}=C^{-1}$, $C^{R_{2}}=A^{-1}, D^{R_{0}}=B C, D^{R_{1}}=D^{-1}$ and $D^{R_{2}}=A^{-1} D^{-1} C$. This group factors $\Delta$ into a dihedral group $D_{3}$ with 6 elements. By the Reidmaster-Schreier Rewriting Process, 
$K_{3}$ is isomorphic to a free product $C_{\infty} * C_{\infty} * C_{\infty} * C_{\infty}$ of rank 4 . Therefore a regular $K_{3^{-}}$ marked hypermap has representative form $\mathcal{Q}=(G ; a, b, c, d)$ where $G$ is a group generated by $a, b, c, d$, without further restrictions. Moreover, as $K_{3}$ is a subgroup of $\Delta^{+}$, any $K_{3^{-}}$ marked hypermap is orientable. The trivial $K_{3}$-hypermap $\mathcal{T}_{K_{3}}$ is the reflexible hypermap $(3,3,3)_{1,0}$ of type $(3,3,3)$ on the torus whose Walsh map is the regular map $\{6,3\}_{1,0}$.

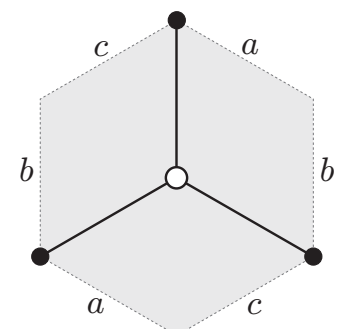

Figure 4: The trivial $K_{3}$-hypermap $\mathcal{T}_{K_{3}}$.

This has 1 hypervertex, 1 hyperedge and 1 hyperface. Therefore the $K_{3}$-type of any regular $K_{3}$-marked hypermap coincides with its topological type (or $\Delta$-type) $(k ; l ; m)$ for some $k=0 \bmod 3, l=0 \bmod 3$ and $m=0 \bmod 3$. By (1.2), $\mathcal{Q}$ has Euler characteristic given by

$$
\chi(\mathcal{Q})=|G|\left(\frac{3}{k}+\frac{3}{l}+\frac{3}{m}-3\right) .
$$

Since

$$
k=\left|r_{1} r_{2}\right|=3\left|\left(r_{1} r_{2}\right)^{3}\right|=3\left|a^{-1} b\right|,
$$

and similarly $l=3\left|c^{-1} d\right|$ and $m=3\left|b c a^{-1} d^{-1}\right|$, we have:

Lemma 2.3. The Euler characteristic of a regular $K_{3}$-marked hypermap $\mathcal{Q}=(G ; a, b$, $c, d)$ is given by

$$
\chi(\mathcal{Q})=|G|\left(\frac{1}{\left|a^{-1} b\right|}+\frac{1}{\left|c^{-1} d\right|}+\frac{1}{\left|b c a^{-1} d^{-1}\right|}-3\right) .
$$

\section{Riemann surfaces}

Let $\mathcal{S}$ be a Riemann surface of genus $g \geq 2$. By the uniformization theorem $S$ is a quotient $\mathbb{U} / \Gamma$, where $\Gamma$ is a cocompact torsion-free discrete subgroup of $\operatorname{Isom}^{+}(\mathbb{U}) \cong \operatorname{PSL}(2, \mathbb{R})$, the group of orientation-preserving isometries of the hyperbolic plane $\mathbb{U}$ (modelled on the complex upper half-plane). The group $\Gamma$, the surface-group corresponding to $S$, is a Fuchsian group with signature $(g ;-)$; it is unique up to a conjugacy in $P S L(2, \mathbb{R})$. Automorphisms of $\mathcal{S}$ lift to isometries of $\mathbb{U}$ normalising $\Gamma$, so if $G$ is a group of automorphisms of $\mathcal{S}$ then $G=\Lambda / \Gamma$, where $\Lambda$ is a Fuchsian group containing $\Gamma$ as a normal subgroup. Let $\left(\lambda ; m_{1}, \ldots, m_{r}\right)$ be the signature of $\Lambda$. This means that $\Lambda$ has presentation

$$
\left\langle x_{1}, \ldots, x_{r}, a_{1}, b_{1}, \ldots, a_{\lambda}, b_{\lambda} \mid x_{1}^{m_{1}}=\ldots=x_{r}^{m_{r}}=\prod_{i=1}^{r} x_{i} \prod_{i=1}^{\lambda}\left[a_{i}, b_{i}\right]=1\right\rangle
$$


This has measure $\mu(\Lambda)=2 \pi\left(2 \lambda-2+\sum_{i=1}^{r}\left(1-\frac{1}{m_{i}}\right)\right)$. The Riemann-Hurwitz formula $\mu(\Gamma)=|\Lambda / \Gamma| \mu(\Lambda)$ can be written as

$$
2 g-2=|G|\left(2 \lambda-2+\sum_{i=1}^{r}\left(1-\frac{1}{m_{i}}\right)\right) \Leftrightarrow \chi(\mathcal{S})=|G|\left(\sum_{i=1}^{r} \frac{1}{m_{i}}-(r+2 \lambda-2)\right)
$$

where $\chi(\mathcal{S})=2-2 g$ and the sum is considered empty in the case $r=0$. Since the order of the periods in the signature is irrelevant, we assume that $m_{1} \leq m_{1} \leq \cdots \leq m_{r}$. Using the same terminology as in [3], we say that a group $G$ acts on genus $g$ with signature $\left(\lambda ; m_{1}, \ldots, m_{r}\right)$ if $G=\Lambda / \Gamma$ where $\Lambda$ and $\Gamma$ are Fuchsian groups such that (1) $\Lambda$ has signature $\left(\lambda ; m_{1}, \ldots, m_{r}\right)$ and (2) $\Gamma$ has signature $(g ;-)$ and is normal in $\Lambda$. In this case $G$ acts on the Riemann surface $\mathcal{S}=\mathbb{U} / \Gamma$ as a group of automorphisms. The canonical epimorphism from $\Lambda$ to $G$ with kernel $\Gamma$ is called a surface-kernel epimorphism. Surfacekernel epimorphisms are order-preserving, so the images of the generators $x_{1}, \ldots, x_{r}$ in $G$ have orders $m_{1}, \ldots, m_{r}$ respectively.

Theorem 3.1. Let $\mathcal{S}$ be a Riemann surface of genus $g>1$ and let $G$ be a group of automorphism of $\mathcal{S}$. If $|G|>g-1$ then $G$ is the automorphism group of a regular restrictedlymarked hypermap $\mathcal{Q}$ of genus $g$. Moreover, $G$ is the automorphism group of a regular $K_{3}$-marked hypermap if $G$ acts with signature $\left(1 ; m_{1}, \ldots, m_{r}\right)$, and of a regular $\Delta_{r-2^{-}}$ marked hypermap if $G$ acts with signature $\left(0 ; m_{1}, \ldots, m_{r}\right)$.

Proof. Let $G=\Lambda / \Gamma$ for some Fuchsian group $\Lambda$ normalising $\Gamma$ (the surface-group) and let $\left(\lambda ; m_{1}, \ldots, m_{r}\right)$ be the signature of $\Lambda$. By the Riemann-Hurwitz formula,

$$
|G|=\frac{2(g-1)}{2 \lambda-2+\sum_{i=1}^{r}\left(1-\frac{1}{m_{i}}\right)} .
$$

Now $|G|>g-1$ implies

$$
0<2 \lambda-2+\sum_{i=1}^{r}\left(1-\frac{1}{m_{i}}\right)<2
$$

and this inequality implies $\lambda \leq 1$.

I : $\lambda=1$. In this case $0<r<4$.

(a) $\mathbf{r}=\mathbf{3}$ Then $\Lambda$ has signature $\left(1 ; m_{1}, m_{2}, m_{3}\right)$ and thus

$$
G=\left\langle x_{1}, x_{2}, x_{3}, a, b \mid x_{1}^{m_{1}}=x_{2}^{m_{2}}=x_{3}^{m_{3}}=x_{1} x_{2} x_{3}[a, b]=1, \ldots\right\rangle .
$$

Fourth relation gives $x_{2}=x_{1}^{-1}[a, b]^{-1} x_{3}^{-1}$. Replacing $x_{2}$ by its inverse we may take $x_{2}=x_{3}[a, b] x_{1}$ to get

$$
G=\left\langle x_{1}, x_{3}, a, b \mid x_{1}^{m_{1}}=x_{3}^{m_{3}}=\left(x_{3}[a, b] x_{1}\right)^{m_{2}}=1, \ldots\right\rangle .
$$

Equation (3.1) can then be written as

$$
\chi(\mathcal{S})=|G|\left(\frac{1}{m_{1}}+\frac{1}{m_{2}}+\frac{1}{m_{3}}-3\right) .
$$


Now we will try to see $G$ as the automorphism group of a regular $K_{3}$-marked hypermap $\mathcal{Q}=(G ; A, B, C, D)$. Taking into account Lemma 2.3, we will try to solve

$$
\left|A^{-1} B\right|=m_{3},\left|C^{-1} D\right|=m_{1} \text { and }\left|B C A^{-1} D^{-1}\right|=m_{2},
$$

knowing that $\left|x_{1}\right|=m_{1},\left|x_{3}\right|=m_{3}$ and $\left|x_{3}[a, b] x_{1}\right|=m_{2}$.

We do this in two steps.

First, since $m_{1}=\left|C^{-1} D\right|=\left|D C^{-1}\right|=\left|C D^{-1}\right|$, we set $C D^{-1}=x_{1}$, from which we get $D=x_{1}^{-1} C$. Then putting $x_{3}[a, b] x_{1}=B C A^{-1} D^{-1}=B C A^{-1} C^{-1} x_{1}$, we get $x_{3}[a, b]=B C A^{-1} C^{-1}$.

Second, since $\left|A^{-1} B\right|=\left|B A^{-1}\right|$, set $B A^{-1}=x_{3}$. This gives $B=x_{3} A$. Replacing $B$ in the above equation we get $[a, b]=A C A^{-1} C^{-1}=\left[A^{-1}, C^{-1}\right]$.

Hence $A=a^{-1}, C=b^{-1}, B=x_{3} A=x_{3} a^{-1}$ and $D=x_{1}^{-1} C=x_{1}^{-1} b^{-1}$ is a solution. Since they generate $G$,

$$
\mathcal{Q}=\left(G ; a^{-1}, x_{3} a^{-1}, b^{-1}, x_{1}^{-1} b^{-1}\right)
$$

is a regular $K_{3}$-marked hypermap with automorphism group $G$ and Euler characteristic

$$
\begin{aligned}
\chi(\mathcal{Q}) & =|G|\left(\frac{1}{\left|A^{-1} B\right|}+\frac{1}{\left|C^{-1} D\right|}+\frac{1}{\left|B C A^{-1} D^{-1}\right|}-3\right) \\
& =|G|\left(\frac{1}{\left|a x_{3} a^{-1}\right|}+\frac{1}{\left|b x_{1}^{-1} b^{-1}\right|}+\frac{1}{\left|x_{3}[a, b] x_{1}\right|}-3\right) \\
& =|G|\left(\frac{1}{m_{3}}+\frac{1}{m_{1}}+\frac{1}{m_{2}}-3\right),
\end{aligned}
$$

which gives $\operatorname{genus}(\mathcal{Q})=g=\operatorname{genus}(\mathcal{S})$.

(b) $\mathbf{r}=\mathbf{2}$ In this case $G=\left\langle x_{1}, x_{2}, a, b \mid x_{1}^{m_{1}}=x_{2}^{m_{2}}=x_{1} x_{2}[a, b]=1, \ldots\right\rangle=\langle x, a, b|$ $\left.x^{m_{1}}=([a, b] x)^{m_{2}}=1, \ldots\right\rangle$. Equation (3.1) takes the form

$$
\chi=|G|\left(\frac{1}{m_{1}}+\frac{1}{m_{2}}-2\right) .
$$

To see $G$ as the automorphism group of a regular $K_{3}$-marked hypermap $\mathcal{Q}=(G ; A$, $B, C, D)$ with $\chi=|G|\left(\frac{1}{\left|A^{-1} B\right|}+\frac{1}{\left|C^{-1} D\right|}+\frac{1}{B C A^{-1} D^{-1} \mid}-3\right)$ we start by putting $A^{-1} B=1$, that is, $B=A$. Comparing formulas we must now have $\left|C^{-1} D\right|=$ $m_{1}$ and $\left|A C A^{-1} D^{-1}\right|=m_{2}$. Following a similar procedure as above, we easily get a solution $A=a^{-1}, B=A=a^{-1}, C=b^{-1}, D=x^{-1} b^{-1}$ and so $\mathcal{Q}=\left(G ; a^{-1}, a^{-1}, b^{-1}, x^{-1} b^{-1}\right)$ ( or $\left.\left(G ; a, a, b, x^{-1} b\right)\right)$ is a regular $K_{3}$-marked hypermap with automorphism group $G$ and Euler characteristic

$$
\chi(\mathcal{Q})=|G|\left(\frac{1}{m_{1}}+\frac{1}{m_{2}}-2\right)=\chi(\mathcal{S}) .
$$

(c) $\mathbf{r}=1$ If $G$ acts with signature $(1 ; m)$ then $G$ has presentation $\langle a, b|[a, b]^{m}=\cdots=$ 1). Expression (3.1) becomes

$$
|G|\left(\frac{1}{m}-1\right)=\chi(\mathcal{S}) .
$$


In this case the regular $K_{3}$-marked hypermap $\mathcal{Q}=(G ; a, a, b, b)$ has Euler characteristic

$$
\chi(\mathcal{Q})=|G|\left(\frac{1}{m}-1\right)=\chi(\mathcal{S}) .
$$

II : $\lambda=\mathbf{0}$. In this case $2<\sum_{i=1}^{r}\left(1-\frac{1}{m_{i}}\right)<4$ implies $2<r<8$.

If $G$ acts with signature $\left(0 ; m_{1}, \ldots, m_{r}\right)$ then $G$ has presentation $\left\langle x_{1}, \ldots, x_{r-1}\right.$ $\left.x_{1}^{m_{1}}=\cdots=x_{r-1}^{m_{r-1}}=\left(x_{1} . x_{r-1}\right)^{m_{r}}=\ldots=1\right\rangle$ with $r-1$ elliptic generators and no parabolic generators. In this case the Riemann-Hurwitz formula yields

$$
\chi(\mathcal{S})=|G|\left(\frac{1}{m_{1}}+\cdots+\frac{1}{m_{r-1}}+\frac{1}{m_{r}}-(r-2)\right) .
$$

It is clear that the (canonical) regular $\Delta_{r-2}$-marked hypermap $\mathcal{Q}=\left(G ; x_{1}, \ldots, x_{r-1}\right)$ will do. The product $x_{r}=x_{1} \ldots x_{r-1}$ has order $m_{r}$ and so by Lemma 2.1,

$$
\chi(\mathcal{Q})=|G|\left(\sum_{i=1}^{r} \frac{1}{m_{i}}-(r-2)\right)=\chi(\mathcal{S})
$$

which shows that $\operatorname{genus}(\mathcal{Q})=g$.

\section{A note on finite marked permutations groups}

Marked finite transitive permutation groups (MFTPG) are triples $\mathfrak{M}=(G, \Omega, D)$ consisting of a finite set $\Omega$ and a set $D=\left\{z_{1}, \ldots, z_{n-1}\right\}$ of permutations of $\Omega$ generating $G$ and acting transitively on $\Omega$ (Singerman [9]). The genus of a marked finite transitive permutation group $\mathfrak{M}$ was defined by Singerman as being the genus of a certain Riemann surface $\mathcal{S}:=\mathbb{X} / N$ "naturally" associated to $\mathfrak{M}$. Although not originally aimed to give an insight on higher dimensional combinatorial structures associated to higher dimensional manifolds, MFTPGs are related with Vince's combinatorial maps [10], or the more general Ferri's connected $(n+1)$-coloured graphs [4]. In fact, any finite combinatorial map $\mathcal{G}$ of rank $I$ is a marked finite transitive permutation group $(G, V(G), D)$ where $V(G)$ is the set of vertices of $\mathcal{G}$ and $D$ consists of $|I|$ fixed-point free involutory permutations of $V(G)$ induced by the $|I|$ edge-colours of $\mathcal{G}$. The transitivity of $G$ on $V(G)$ is a consequence of the connectivity of $\mathcal{G}$ and the fixed-point free action of the involutory permutations a consequence of the non-existence of free edges in $\mathcal{G}$ (when realised as cell decompositions this corresponds to boundary-free manifolds). These were all designed to describe cell decompositions of $n$-dimensional manifolds, $n$-polytopes and tessellations $(n=|I|-1)$ ), though in general not all such combinatorial constructions of rank $>3$ are realised in such a way. Despite the name, a combinatorial map is just a graph and when its degree (rank) is 3 it actually describes a 2-dimensional simplicial complex (best known as a hypermap).

In the special case when $D=\left\{z_{1}, z_{2}\right\}$ and $z_{1}$ is a fixed-point free involution, which corresponds to an oriented map, Singerman showed that the associated "Riemann" genus coincide with the genus of the map. In general Singerman did not associate MFTPGs to embeddings of graphs on surfaces (of genus $g$ ), except in the above mentioned case. We can now show that actually any marked finite transitive permutation group $\mathfrak{M}$ of genus $g$ represents a restrictedly-marked hypermap $\mathcal{Q}$ of genus $g$. These "coloured" maps representing restrictedly-marked hypermaps are not related with Vince's and Ferri's edge-coloured graphs. 
Let $\mathfrak{M}=(G, \Omega, D)$ be a marked finite transitive permutation group with $D=\left\{z_{1}, \ldots\right.$, $\left.z_{n-1}\right\}$. If $\operatorname{rank}(\mathfrak{M})=|D|=1$ then $G$ is cyclic generated by one element and by the transitivity of $G$ on $\Omega$ we must have $|\Omega|=|G|$. So $\mathfrak{M}$ is a regular oriented ( $\Delta^{+}$-restricted) $\operatorname{map} \mathcal{M}=\left(G ; z_{1}, 1\right)$.

For the rest of the paper let $\operatorname{rank}(\mathfrak{M})>1(n>2)$. Let $k_{1}, \ldots, k_{n-2}$ be the orders of $z_{1}, \ldots, z_{n-2}$ respectively, $l:=n\left|z_{n-1}\right|$ and $m:=n\left|z_{n}\right|$, where $z_{n}=z_{1} \ldots z_{n-1}$. Let $\Gamma$ be a F-group with signature $\left(0 ; k_{1}, \ldots, k_{n-2}, \frac{l}{n}, \frac{m}{n}\right)$; this means that $\Gamma$ has presentation

$$
\left\langle x_{1}, \ldots, x_{n-2}, x_{n-1}, x_{n} \mid x_{1}^{k_{1}}=\cdots=x_{n-2}^{k_{n-2}}=x_{n-1}^{\frac{l}{n}}=x_{n}^{\frac{m}{n}}=\prod_{i=1}^{n} x_{i}=1\right\rangle .
$$

This has measure given by $\mu(\Gamma)=2 \pi\left(n-2-\left(\sum_{i=1}^{n-2} \frac{1}{k_{i}}+\frac{n}{l}+\frac{n}{m}\right)\right)$. We have an obvious epimorphism $\varrho: \Gamma \rightarrow G$ defined by $x_{i} \mapsto z_{i}$ for $i=1, \ldots, n-1$. Let $N=\operatorname{Stab}_{G}(w) \varrho^{-1} \triangleleft_{q} \Gamma$, where $q=|\Theta: Q|=|\Omega|$. This group $N$ is also a F-group and $\mathcal{S}:=\mathbb{X} / N$ is a Riemann surface, where $\mathbb{X}$ is the sphere $\mathbb{S}$ if $\mu(\Gamma)<0$, the Euclidean plane $\mathbb{C}$ if $\mu(\Gamma)=0$ or the hyperbolic plane $\mathbb{H}$ if $\mu(\Gamma)>0$ (Singerman [9]). Singerman defined the genus of $\mathfrak{M}$ to be the genus of the Riemann surface $\mathcal{S}$. On the other hand, $\mathfrak{M}$ corresponds to an orientable $\Delta_{n-2}$-marked hypermap $\mathcal{Q}=\left(\Omega, z_{1}, \ldots, z_{n-1}\right)$, where $\Delta_{n-2}=\left\langle Z_{1}, \ldots, Z_{n-1}\right\rangle \triangleleft_{2(n-2)} \Delta$ is the group generated by $Z_{1}=R_{1} R_{2}, Z_{2}=$ $\left(R_{1} R_{2}\right)^{R_{0} R_{2}}, \ldots, Z_{n-2}=\left(R_{1} R_{2}\right)^{\left(R_{0} R_{2}\right)^{n-3}}$ and $Z_{n-1}=\left(R_{2} R_{0}\right)^{n-2}$. Let $Z_{n}$ be the product $Z_{1} \ldots Z_{n-1}=\left(R_{1} R_{0}\right)^{n-2}$ and $Q$ its $\Delta_{n-2}$-marked fundamental subgroup - The orientable restricted marked hypermap $\mathcal{Q}$ has genus $g(\mathcal{Q})=\frac{2-\chi}{2}$ where $\chi=$ $|\mathcal{V}|+|\mathcal{E}|+|\mathcal{F}|-\frac{|F|}{2}$ is the Euler characteristic of the underlying surface ( $F$ is the set of flags of its $\Delta$-form ${ }^{\Delta} \mathcal{Q}$ ). Although natural, it is not yet clear whether $\mathcal{Q}$ has genus $g$ (see theorem 6), since different $\Theta$-marked hypermaps can be associated to a given group, with different genus.

Theorem 4.1. A marked finite permutation group $\mathfrak{M}=(G, \Omega, D)$, where $D=\left\{z_{1}, \ldots\right.$, $\left.z_{n-1}\right\}$, is an orientable $\Delta_{n-2}$-marked hypermap $\mathcal{Q}=\left(\Omega, z_{1}, \ldots, z_{n-1}\right)$ with genus $(\mathcal{Q})=$ $\operatorname{genus}(\mathfrak{M})$.

Proof. Let $g=\operatorname{genus}(\mathfrak{M})$. Decompose $z_{1}, \ldots, z_{n}$ in a product of cycles:

$$
\begin{aligned}
& z_{1}=\sigma_{1,1} \cdots \sigma_{1, t_{1}} \\
& \cdots \\
& z_{n-2}=\sigma_{n-2,1} \cdots \sigma_{n-2, t_{n-2}} \\
& z_{n-1}=\alpha_{1} \cdots \alpha_{s} \\
& z_{n}=\beta_{1} \cdots \beta_{r} .
\end{aligned}
$$

Then the number of hypervertices $|\mathcal{V}|$, the number of hyperedges $|\mathcal{E}|$ and the number of hyperfaces $|\mathcal{F}|$ are given by $|\mathcal{V}|=\sum_{i-1}^{n-2} t_{i},|\mathcal{E}|=s$ and $|\mathcal{F}|=r$, respectively. The number of flags $|\Omega|=|\Theta: Q|=q$ is related to the above cycle decomposition as

$$
|\Omega|=\sum_{j=1}^{t_{i}}\left|\sigma_{i, j}\right|=\sum_{i=1}^{s}\left|\alpha_{i}\right|=\sum_{i=1}^{r}\left|\beta_{i}\right| .
$$

Now the F-group $N$ has signature (Singerman [9])

$$
\left(g ; \frac{k_{1}}{\delta_{1,1}}, \ldots, \frac{k_{1}}{\delta_{1, t_{1}}}, \ldots, \frac{k_{n-2}}{\delta_{n-2,1}}, \ldots, \frac{k_{n-2}}{\delta_{n-2, t_{n-2}}}, \frac{\frac{l}{n}}{a_{1}}, \ldots, \frac{\frac{l}{n}}{a_{s}}, \frac{\frac{m}{n}}{b_{1}}, \ldots, \frac{\frac{m}{n}}{b_{r}}\right),
$$


where $\delta_{i, j}=\left|\sigma_{i, j}\right|, a_{i}=\left|\alpha_{i}\right|$ and $b_{i}=\left|\beta_{i}\right|$. The measures of the F-groups $N$ and $\Gamma$ are related by $\mu(N)=|\Omega| \mu(\Gamma)$. This formula translates as

$$
\begin{array}{r}
2 g-2+\sum_{i=1}^{t_{1}}\left(1-\frac{\delta_{1, i}}{k_{1}}\right)+\cdots+\sum_{i=1}^{t_{n-2}}\left(1-\frac{\delta_{n-2, i}}{k_{n-2}}\right)+\sum_{i=1}^{s}\left(1-\frac{a_{i}}{\frac{l}{n}}\right)+\sum_{i=1}^{r}\left(1-\frac{b_{i}}{\frac{m}{n}}\right)= \\
|\Omega|\left(n-2-\sum_{i=1}^{n-2} \frac{1}{k_{i}}-\frac{n}{l}-\frac{n}{m}\right) .
\end{array}
$$

Since $\sum_{j=1}^{t_{i}} \delta_{i, j}=|\Omega|, \sum_{i=1}^{s} a_{i}=|\Omega|$ and $\sum_{i=1}^{r} b_{i}=|\Omega|$, we get

$2 g-2+\sum_{i=1}^{n-2} t_{i}+s+r-\frac{|\Omega|}{k_{1}}-\cdots-\frac{|\Omega|}{k_{n-2}}-\frac{|\Omega|}{\frac{l}{n}}-\frac{|\Omega|}{\frac{m}{n}}=|\Omega|(n-2)-\sum_{i=1}^{n-2} \frac{|\Omega|}{k_{i}}-|\Omega|\left(\frac{n}{l}+\frac{n}{m}\right)$.

Now replacing $\sum_{i=1}^{n-2} t_{i}$ by $|\mathcal{V}|, s$ by $|\mathcal{E}|, r$ by $|\mathcal{F}|$ and taking into account that $\left|\Delta: \Delta_{n}\right|=$ $2(n-2)$, and thus $|\Omega| 2(n-2)=|\Theta: Q||\Delta: \Theta|=|\Delta: Q|=|F|$, we get

$$
|\mathcal{V}|+|\mathcal{E}|+|\mathcal{F}|-\frac{|F|}{2}=2-2 g
$$

Thus $\chi(\mathcal{Q})=2-2 g$ and hence $\operatorname{genus}(\mathcal{Q})=g$.

Any hypermap $\mathcal{H}$ is a $\Delta$-marked hypermap. As $\mathcal{H}$ may also be represented by some $\Theta$-marked hypermap $\mathcal{Q}$, with flags in $\mathcal{H}$ being represented by $\Theta$-slices in $\mathcal{Q}$, the genus of the $\Delta$-marked hypermap $\mathcal{H}$ may be different from the genus of the $\Theta$-marked hypermap $\mathcal{Q}$. Theorem 4.1 just emphasises how natural is the choice of $\Delta_{n}$ in the restrictedly-marked subgroups considered earlier.

\section{Acknowledgment}

I would like to express my gratitude to the referees for spotting a mistake in an earlier version of the paper.

\section{References}

[1] A. Breda d'Azevedo, A theory of restricted regularity of hypermaps, J. Korean Math. Soc. 43 (2006), 991-1018.

[2] A. Breda d'Azevedo and G. Jones, Double coverings and reflexible abelian hypermaps, Beitr. Algebra Geom. 41 (2000), 371-389.

[3] E. Bujalance, F. J. Cirre and M. D. E. Conder, On extendability of group actions on compact Riemann surfaces, Trans. Amer. Math. Soc. 355 (2003), 1537-1557.

[4] M. Ferri, Una rappresentazione delle $n$-varietà topologiche triangolabili mediante grafi $(n+1)$ colorati, Boll. Un. Mat. Ital. 13-B (1976), 250-260.

[5] A. Hurwitz, Über algebraische Gebilde mit eindeutige Transformationen in sich, Math. Ann. 41 (1893), 403-442.

[6] G. A. Jones and D. Singerman, Theory of maps on orientable surfaces, Proc. London Math. Soc. 37 (1978), 273-307. 
[7] W. Magnus, A. Karrass and D. Solitar,Combinatorial Group Theory, Dover Books, New York, 1976.

[8] D. Singerman, Symmetries of Riemann surfaces with large automorphism group, Math. Ann. 210 (1974), 17-32.

[9] D. Singerman, Automorphisms of maps, permutations groups and Riemann surfaces, Bull. Amer. Math. Soc. 8 (1976), 65-68.

[10] A. Vince, Combinatorial maps, J. Combin. Theory Ser. B 34 (1983), 1-21. 